\title{
Cerebral Gaseous Microemboli are Detectable During Continuous Venovenous Hemodialysis in Critically III Patients: An Observational Pilot Study
}

\author{
Gabor Erdoes, MD,* $\dagger$ Clemens Kietaibl, MD, ${ }^{*}$ Stefan Boehme, MD,* Roman Ullrich, MD,* \\ Klaus Markstaller, MD, ${ }^{*}$ Balthasar Eberle, MD, $\dagger$ and Klaus U. Klein, MD*
}

\begin{abstract}
Background: Continuous venovenous hemodialysis (CVVHD) may generate microemboli that cross the pulmonary circulation and reach the brain. The aim of the present study was to quantify (load per time interval) and qualify (gaseous vs. solid) cerebral microemboli (CME), detected as high-intensity transient signals, using transcranial Doppler ultrasound.
\end{abstract}

Materials and Methods: Twenty intensive care unit (ICU group) patients requiring CVVHD were examined. CME were recorded in both middle cerebral arteries for 30 minutes during CVVHD and a CVVHD-free interval. Twenty additional patients, hospitalized for orthopedic surgery, served as a non-ICU control group. Statistical analyses were performed using the MannWhitney $U$ test or the Wilcoxon matched-pairs signed-rank test, followed by Bonferroni corrections for multiple comparisons.

Results: In the non-ICU group, 48 (14.5-169.5) (median [range]) gaseous CME were detected. In the ICU group, the 67.5 (14.5588.5) gaseous CME detected during the CVVHD-free interval increased 5-fold to 344.5 (59-1019) during CVVHD $(P<0.001)$. The number of solid CME was low in all groups (non-ICU group: 2 [0-5.5]; ICU group CVVHD-free interval: 1.5 [0-14.25]; ICU group during CVVHD: 7 [3-27.75]).

Conclusions: This observational pilot study shows that CVVHD was associated with a higher gaseous but not solid CME burden in critically ill patients. Although the differentiation between

Received for publication November 8, 2015; accepted February 16, 2016. From the *Department of Anesthesia, General Intensive Care and Pain Management, Medical University of Vienna, Wien, Austria; and $\dagger$ Department of Anesthesiology and Pain Therapy, University Hospital Bern, Bern, Switzerland.

G.E., K.U.K., K.M., R.U., and B.E.: planned the study. G.E. and K.U.K.: performed the experiments in the ICU group, and C.K. and K.U.K: performed the experiments in the non-ICU control group. G.E., C.K., S.B., and K.U.K.: conducted the data analysis. All authors read and approved the final manuscript.

Part of this study was presented as a poster at the Austrian International Congress of Anesthesiology, Linz, on November 28, 2014 and won the third poster prize.

Supported by the Department of Anesthesia, General Intensive Care and Pain Management, Medical University of Vienna, Austria.

The authors have no conflicts of interest to disclose.

Reprints: Klaus U. Klein, MD, Department of Anesthesia, General Intensive Care and Pain Management, Medical University of Vienna, Waehringer Guertel 18-20, Vienna A-1090, Austria (e-mail: ulrich. klein@meduniwien.ac.at).

Copyright (C) 2016 Wolters Kluwer Health, Inc. All rights reserved. gaseous and solid CME remains challenging, our finding may support the hypothesis of microbubble generation in the CVVHD circuit and its transpulmonary translocation toward the intracranial circulation. Importantly, the impact of gaseous and solid CME generated during CVVHD on brain integrity of critically ill patients currently remains unknown and is highly debated.

Key Words: transcranial Doppler ultrasound, high-intensity transient signals, continuous venovenous hemodialysis, cerebral microemboli

(J Neurosurg Anesthesiol 2016;00:000-000)

A far back as 1975 it was postulated that in patients undergoing hemodialysis, microemboli are generated that enter the pulmonary circulation and cause pulmonary inflammation and injury. ${ }^{1}$ Subsequently, the presence of microemboli has been repeatedly demonstrated downstream of the arteriovenous fistula, the subclavian vein, and the venous air trap and outlet line of the hemodialysis circuit. ${ }^{2-7}$ Microemboli that enter the cardiovascular system can cause deleterious effects in the lungs and remote organs, including microembolization as well as activation of coagulation, complement, leukocytes, and platelets. ${ }^{8}$ Importantly, the burden of microemboli that forms during chronic hemodialysis appears significant, with potentially $>500$ microemboli per minute generated in the hemodialysis circuit, which may bypass the air trap and enter the venous circulation. ${ }^{7}$

Recent research suggested that the microemboli that develop during hemodialysis may pass through the lung barrier and that they can be detected in the carotid artery using Doppler ultrasound scanning. ${ }^{9}$ It seems possible that microemboli may cause injury to remote organs such as the brain. Patients with chronic kidney disease that require hemodialysis experience worse cognitive outcomes than age-matched and sex-matched control individuals, supporting the possibility that microemboli may contribute to brain dysfunction. ${ }^{10}$ Like solid microemboli, gaseous microemboli that form into larger gaseous bubbles can cause capillary occlusion and hypoxia downstream of the blockade. According to the Epstein-Plesset model, the dissolution time for a $1 \mathrm{~mm}^{3}$ air bubble is in 
the range of 11 to 70 days, highlighting the potential danger of gaseous microemboli. ${ }^{8}$ Modern transcranial Doppler ultrasound (TCD) allows for the detection and differentiation of high-intensity transient signals (HITS) in both middle cerebral arteries (MCAs) into artefacts, solid, and gaseous cerebral microemboli (CME) with high accuracy. Emboli differentiation is based on the fact that solid microemboli reflect ultrasound at lower frequencies and decibel ranges compared with gaseous microemboli. ${ }^{11}$ However, there are currently no published articles reporting CME counts in critically ill patients undergoing continuous venovenous hemodialysis (CVVHD).

The aim of the present study was to use multifrequency pulsed TCD to both quantify (count per $30 \mathrm{~min}$ ) and characterize (gaseous vs. solid) CME in both MCAs in intensive care unit (ICU) patients during running and connected (activated) CVVHD and during a CVVHD-free interval. We hypothesized that gaseous and solid CME counts would increase during activated CVVHD as compared with those during CVVHD-free interval. In addition, TCD measurements were performed in hospitalized orthopedic patients without CVVHD therapy that served as a non-ICU control group.

\section{MATERIALS AND METHODS}

\section{Study Design}

This study included an ICU group and a non-ICU group. For the ICU group, the study design was a singlecenter, prospective, observational, pilot clinical trial. The research was conducted in agreement with the Declaration of Helsinki. Ethical approval was provided by the Ethics Committee of the Medical University of Vienna, Austria (No. 1706/2013, Chair: E. Singer) on September 30,2013 . The study was registered with the United States National Institutes of Health (US NIH, ClinicalTrials.gov \#NCT02021045) in November 2013. All procedures were performed at the Medical University of Vienna between September 2013 and January 2014 after obtaining written patient informed consent. The patient cohort consisted of awake and sedated ICU patients $(\mathrm{n}=20)$ aged between 18 and 90 years undergoing CVVHD. The study inclusion criterion was CVVHD renal replacement therapy performed in the in-house ICU. The study exclusion criteria were having a preexisting neurological morbidity, neurocognitive disturbances, carotid artery stenosis $>70 \%$, medical history of patent foramen ovale, treatment with other extracorporeal devices (such as extracorporeal membrane oxygenation), cardiac pathologies (valvular heart disease, endocarditis), pregnancy, drug abuse, an allergy to any materials contained in the medical device used in the study, or temporal skin/bone lesions.

The non-ICU group was added post hoc because the ICU group showed significant gaseous CME counts during the CVVHD-free time interval. This control group consisted of non-ICU patients from another ongoing clinical study (the first 20 consecutive patients with bilateral TCD signals) that were included in our data analysis to allow us a better interpretation of the findings in the ICU population. This second study was a singlecenter, prospective, observational, clinical trial. The research was conducted in agreement with the Declaration of Helsinki. Ethical approval was provided by the Ethics Committee of the Medical University of Vienna, Austria (No. 1633/2013, Chair: E. Singer) on September 09, 2013. The study was registered with the US NIH (ClinicalTrials.gov \#NCT02340416) in January 2015. All procedures were performed at the Medical University of Vienna between August 2014 and November 2014 after obtaining written patient informed consent. The patient cohort consisted of awake patients aged between 50 and 90 years, undergoing a baseline TCD examination before elective orthopedic surgery $(n=20)$. The study inclusion criteria were implantation of a hip/knee/shoulder prosthesis, or spinal surgery. The study exclusion criteria were presence of a severe valvular heart defect or an acute neurological disorder.

\section{Protocol}

The ICU group patients were enrolled in the study by G.E. or K.U.K. in chronological order, according to their initiation of CVVHD in the ICU. All patients were subject to standard ICU monitoring (Dräger Infinity Delta, Lübeck, Germany). All patients were placed in the routine ICU positioning supine with 30 -degree elevation of the head. All patients received standard ICU treatment according to the individual patient needs. All lines from and to the patients were checked for correct closed connections and correct placement. Each patient was measured first during CVVHD therapy for 30 minutes and thereafter during the CVVHD-free interval for 30 minutes. The study protocol required stopping of the CVVHD therapy and disconnecting the circuit tubing connections, therefore, a recovery period of 30 minutes was maintained between both TCD examinations. No other interventions including drug injections, line flushing, changing the perfusor infusion rate, changing the respirator setting, body or head movement or any other treatments were permitted during the examinations. For the non-ICU group, 20 awake, hospitalized orthopedic patients not undergoing CVVHD were monitored using the same TCD technology during a TCD baseline examination for a duration of 15 minutes, with data normalized for 30 minutes, before orthopedic surgery (performed by C.K. and K.U.K). All patients were subject to standard operation room monitoring (Dräger Infinity Delta). Likewise, patients were placed supine with 30-degree elevation of the head and no interventions (including drug injections, line flushing, changing the infusion rate, blood infusion, and therapeutic manipulations) were permitted during the TCD examinations.

\section{CVVHD Therapy}

ICU group CVVHD was performed using multiFiltrate (Fresenius Medical Care Deutschland $\mathrm{GmbH}$, Bad Homburg, Germany) according to the manufacturer's specifications. The hemodialysis setup included 
an Ultraflux AV1000S hemofilter, a hemofiltration solution (Ci-Ca dialysate K2 solution), and a high-flow lumen catheter $(14 \mathrm{Fr}, 15 \mathrm{~cm})$ for central venous access (Teleflex Medical; Arrow, NC). Regional anticoagulation was achieved by prefilter infusion of citrate $(\mathrm{CiCa})$ with a target citrate concentration of 3 to $5 \mathrm{mmol} / \mathrm{L}$ in the filter and a postfilter concentration of ionized calcium between 0.2 and $0.4 \mathrm{mmol} / \mathrm{L}$. The multiFiltrate was equipped with a regular air trap and detector using the ultrasound principle (LP 450-3). Although the blood flow (80 to $120 \mathrm{~mL} / \mathrm{min}$ ) and dialysate flow (1500 to $4000 \mathrm{~mL} / \mathrm{min}$ ) of the CVVHD machine were individually set, each setting remained constant during both TCD measurements.

\section{Multifrequency Pulsed TCD Ultrasound}

This study used multifrequency pulsed TCD technology for the real-time detection and differentiation of HITS into artifacts, solid CME, and gaseous CME (Doppler BoxX; Compumedics Germany GmbH, DWL, Singen, Germany). The software is based on the algorithm described by Devuyst and colleagues and uses 4 specific features for the differentiation of artefact and solid and gaseous CME: the time delay, the peak frequency, the relative power, and the symmetry of the signal envelope. ${ }^{12-20}$ Measurements were performed by 2 investigators (G.E. and K.U.K.) according to the guidelines set by the International Consensus Group on HITS detection. ${ }^{21}$ The preparation for the TCD examination requires an average of 15 to 30 minutes to mount the head frame and to locate the optimum bone window area. A size-adjustable probe holder was mounted bilaterally over the temporal bone window for 2.0 and $2.5 \mathrm{MHz}$ MCAs insonation at 55 to $70 \mathrm{~mm}$ depth with a sample volume length of $8 \mathrm{~mm}$ (DiaMon; Compumedics Germany $\mathrm{GmbH}$ ). The pulse repetition frequency was set at $7 \mathrm{kHz}$ and the detection threshold for HITS was adjusted to $9 \mathrm{~dB}{ }^{21}$ Power output and gain settings were adjusted to provide an optimal signal-to-noise ratio. Doppler velocity and power M-mode spectrograms were recorded and the fast Fourier transform size was set at $100 \mathrm{~Hz}$ to allow for real-time signal detection and differentiation (Fig. 1). ${ }^{11}$

\section{Statistics}

Data were analyzed for normality distribution using the Shapiro-Wilk test. The Mann-Whitney $U$ test was used to test for differences between the ICU and non-ICU control groups, followed by the Bonferroni method for multiple comparisons $(P<0.05)$. The Wilcoxon matchedpairs signed-rank test was used to detect differences between CVVHD and the CVVHD-free interval, followed by the Bonferroni method for multiple comparisons $(P<0.05)$. All data are presented as median and interquartile range ( $25 \%$ to $75 \%$ percentile). GraphPad Prism Version 6.0 was used for all data analyses and to produce box and whisker plots (Tukey) and staggered outliers (GraphPad Software, La Jolla, CA).

\section{RESULTS}

\section{Patient Characteristics}

In total, 29 ICU patients were enrolled in the study, and 20 patients ( 6 females and 14 males, aged 29 to $75 \mathrm{y}$ ) were included in the final analysis (Table 1). TCD measurements could not be performed in 5 patients because of the absence of a temporal bone window, and 4 patients were excluded from the final analysis because of premature TCD signal loss due to agitation. CVVHD was initiated because of electrolyte and metabolic disturbances $(\mathrm{n}=15)$, volume overload and concomitant respiratory failure $(n=3)$, or acute kidney failure $(n=2)$. Twenty-five awake, non-ICU control patients scheduled for orthopedic surgery (TCD examination shortly before surgery) were enrolled in the study; the first consecutive 20 patients (12 female, 8 male; aged 51 to $83 \mathrm{y}$ ) with knee surgery $(\mathrm{n}=7)$, hip surgery $(\mathrm{n}=9)$, and spine and shoulder surgery (each $n=2$ ) were included in the final analysis. Five patients were excluded because of the absence of a temporal bone window.

\section{Gaseous and Solid CME}

In the non-ICU group, 48 (14.5 to 169.5) gaseous CME were detected. In the ICU group, there were 67.5 (14.5 to 588.5) gaseous CME during the CVVHD-free interval and 344.5 (59 to 1019) during CVVHD (Fig. 2). A significant difference in gaseous CME counts was detected between the non-ICU control group and the ICU group during CVVHD $(P=0.041)$ and within the ICU group between CVVHD and CVVHD-free interval $(P<0.001)$. No differences in gaseous CME counts were detected between the non-ICU control group and the ICU group during the CVVHD-free interval. Solid CME were low in number and showed a skewed distribution in all groups (non-ICU control group: 2 [0 to 5.5]; ICU group CVVHD-free interval: 1.5 [0 to 14.25]; and ICU group during CVVHD: 7 [3 to 27.75]). A significant difference was only observed between the non-ICU control group and the ICU group during CVVHD therapy $(P=0.033)$.

\section{DISCUSSION}

In this observational pilot study, multifrequency pulsed TCD technology was used to quantify (count per $30 \mathrm{~min}$ ) and qualify (gaseous vs. solid) CME in both MCAs in an ICU group during CVVHD and during a CVVHD-free interval; and in a non-ICU control group of hospitalized orthopedic patients. These study results showed that (a) critically ill patients experience $>5$-fold increase in gaseous but not solid CME counts during activated CVVHD as compared with those during CVVHD-free interval; and (b) also the non-ICU control group showed a significant gaseous CME count.

\section{Origin, Composition, and Transmission of Microemboli}

Numerous in vitro and in vivo studies have confirmed that microemboli are generated in hemodialysis systems that pass through the bubble detection system 


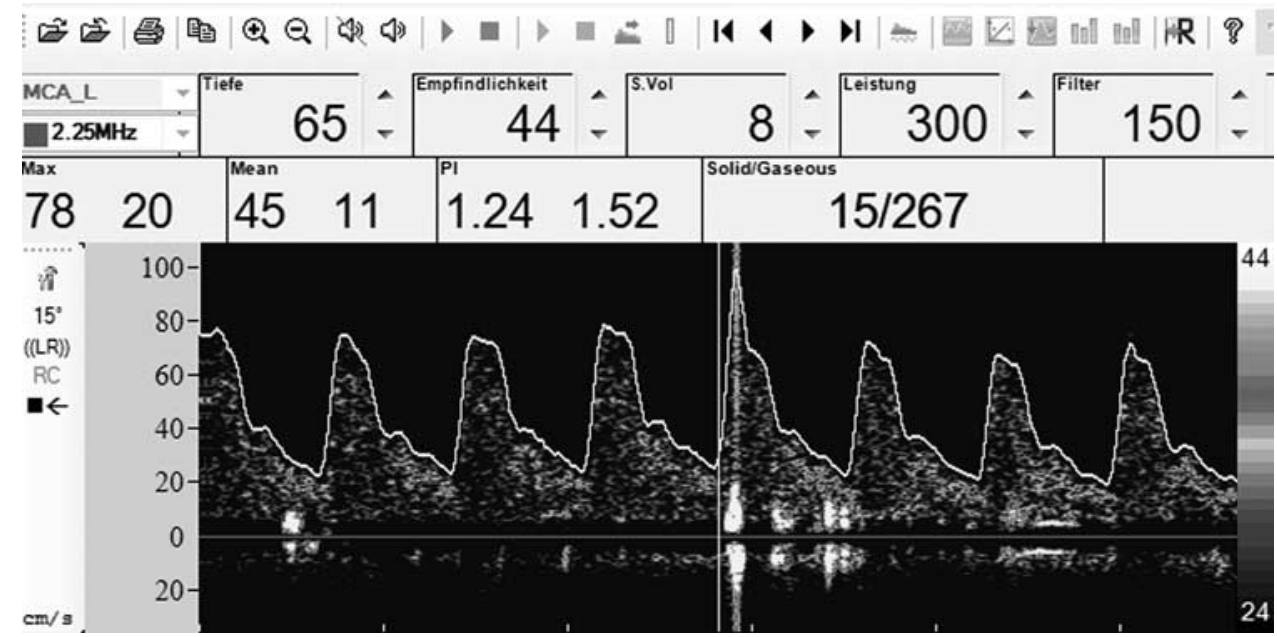

FIGURE 1. Multifrequency-pulsed TCD technology. This technology uses real-time software to detect high-intensity transient signals and can differentiate between solid and gaseous CME. The small light spots in the Doppler signal represent solid CME; gaseous CME cannot be identified visually. CME indicates cerebral microemboli; MCA, middle cerebral artery; TCD, transcranial Doppler ultrasound.

without triggering an alarm..$^{2-7}$ Microemboli caused by hemodialysis are composed of fibrinogen, artificial materials, or air bubbles. ${ }^{8}$ It has been suggested that the majority of microemboli are gaseous in nature based on experimental studies demonstrating that microemboli were still detected in hemodialysis circuits when dextran was used instead of whole blood. ${ }^{22}$ Microemboli that enter the venous circulation reach the lungs and were thought to be filtered in the 5 to $8 \mu \mathrm{m}$ diameter pulmonary capillaries. However, several studies have demonstrated that microemboli can pass the heart or lungs and cause

TABLE 1. Patients' Demographic and Procedural Data

\begin{tabular}{|c|c|c|}
\hline & $\begin{array}{l}\text { ICU Group } \\
(\mathbf{n}=\mathbf{2 0})\end{array}$ & $\begin{array}{l}\text { Non-ICU Group } \\
\quad(\mathbf{n}=\mathbf{2 0})\end{array}$ \\
\hline Age (median [IQR]) (y) & $55(48-66)$ & $69(57-75)$ \\
\hline Female, male (n) & 6,14 & 12,8 \\
\hline \multicolumn{3}{|l|}{ Reason for admission (n [\%]) } \\
\hline Orthopedic surgery & $0(0)$ & $20(100)$ \\
\hline $\begin{array}{l}\text { Abdominal/vascular } \\
\text { surgery }\end{array}$ & $6(30)$ & NA \\
\hline Cardiac/thoracic surgery & $4(20)$ & NA \\
\hline Lung/liver transplant & $5(25)$ & NA \\
\hline Respiratory failure & $3(15)$ & NA \\
\hline Sepsis & $2(10)$ & NA \\
\hline \multicolumn{3}{|l|}{ Indication for CVVHD (n [\%]) } \\
\hline Acute renal failure & $14(70)$ & NA \\
\hline Chronic renal failure & $6(30)$ & NA \\
\hline $\begin{array}{l}\text { Mechanical ventilation (n } \\
[\%])\end{array}$ & $12(60)$ & NA \\
\hline $\begin{array}{l}\text { SAPS3 (points) (median } \\
\text { [IQR]) }\end{array}$ & $49(36-55)$ & NA \\
\hline $\begin{array}{l}\text { Probability of death (median } \\
{[\text { [QR }] \text { ) }}\end{array}$ & $12(3-20)$ & NA \\
\hline
\end{tabular}

Data are presented as median with IQRs (25th and 75th percentile), percent $(\%)$, or number of observations (n).

CVVHD indicates continuous venovenous hemodialysis; ICU, intensive care unit; IQR, interquartile range; NA, not applicable; SAPS3, simplified acute physiology score. arterial embolism. ${ }^{8}$ Notably, $25 \%$ of the general population has a patent foramen ovale that may open during increased thoracic pressure. Transpulmonary passage of microemboli alternatively may be attributed to elevated pulmonary pressure. ${ }^{23}$ Using TCD technology (Emex-25; Hatteland Instrumentering, Royken, Sweden) in patients with renal replacement therapy, Forsberg et $\mathrm{al}^{9}$ demonstrated that microemboli, developed during chronic hemodialysis, passed the lung barrier and could be detected in the carotid arteries. However, transmission of microemboli from the venous circulation to the cerebral circulation could not be confirmed in a study by George et $\mathrm{al}^{24}$ that used another TCD technology (Spencer Technologies, Seattle, WA) for monitoring of CME in the MCAs. However, this finding was surprising as the authors expected detection of CME at least in the patients with open foramen ovale. Both studies are not absolutely contradictory, revealing that microemboli were generated in hemodialysis circuits. Clearly, more scientific data are needed to clarify in which cases microemboli are transmitted to the brain. ${ }^{24}$

\section{Gaseous and Solid CME in the MCAs}

In our study the highest gaseous CME burden was detected during activated CVVHD. This finding is in agreement with the study by Forsberg et $\mathrm{al}^{9}$ who provided first evidence of the transmission of microemboli generated during hemodialysis to the cerebral circulation. As an unexpected finding we also observed a lower but significant count of gaseous CME during the CVVHD-free interval. Possible explanations for this interesting finding include atherosclerotic disease (eg, carotids and proximal aorta) and stagnant or turbulent blood flow (eg, valvular heart disease, intracranial vessel stenosis, presence of intravascular catheters, or intravenous fluid therapy in critically ill patients). ${ }^{25-28}$ Therefore, we decided to include a further control group consisting of non-ICU 
A

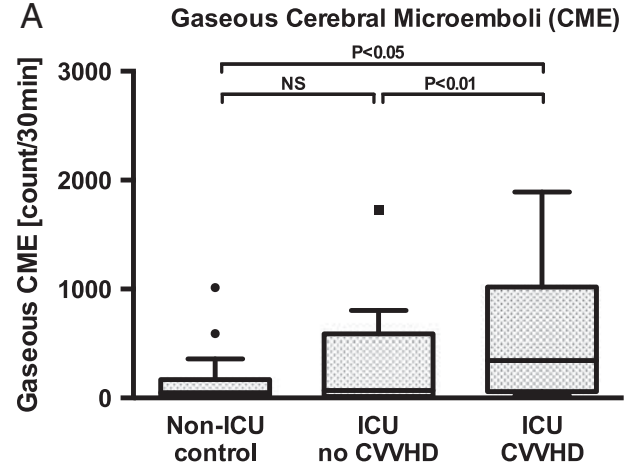

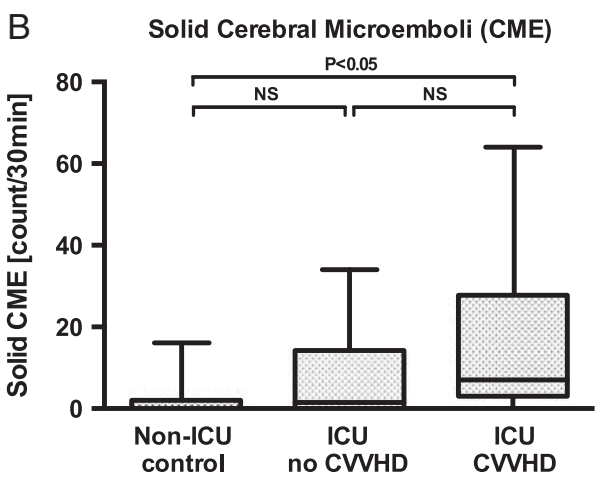

FIGURE 2. Gaseous and solid CME detection. The number of gaseous (A) and solid (B) CME detected with TCD per 30 minutes in both middle cerebral arteries in non-ICU patients $(n=20)$ and in ICU patients $(n=20)$ measured during CVVHD and CVVHD-free time interval (no CVVHD). CVVHD was associated with a higher burden of gaseous but not solid CME than those during CVVHDfree interval in critically ill patients. CME indicates cerebral microemboli; CVVHD, continuous venovenous hemodialysis; ICU, intensive care unit; HITS, high-intensity transient signals; TCD, transcranial Doppler ultrasound.

hospitalized orthopedic patients that did not require ICU therapy and CVVHD treatment. Although a large body of evidence shows that CME are frequently generated during orthopedic surgery, no study has investigated CME count before orthopedic surgery such as performed in the present study. However, other research supports the presence of CME in these cohorts of patients, as CME frequently occur in elderly patients with atherosclerotic aortic or carotid disease, or atrial fibrillation. Recently, it has been suggested that CME in elderly patients might cause structural brain changes and cognitive decline likely attributed to silent brain infarction. ${ }^{29}$

We also observed a higher solid CME count in the ICU group during activated CVVHD than that during the CVVHD-free interval, but this difference did not reach significance. A larger patient cohort might help establish whether CVVHD also independently increases patient solid CME burden. Although it has been proposed that solid CME might cause more harm to the brain than gaseous (and potentially dissolvable) CME, this hypothesis remains unproven at present, as to the best of our knowledge no studies have linked the quality (gaseous vs. solid) of CME to the neurological outcomes of critically ill patients. Both solid and gaseous CME in theory may cause harm to the brain as shown in patients undergoing cardiac surgery. ${ }^{30,31}$ Cerebral ischemia may occur when the number of microemboli exceeds a threshold CME count. Such a threshold for injury has not been established but might presumably differ between individual patients and between numbers of gaseous or solid CME. ${ }^{32}$ In summary, the research on CME evaluation is a difficult but emerging field of study with potential links to silent brain infarctions associated with premature cerebral dysfunction, including dementia. ${ }^{33-36}$

\section{Technological Considerations}

There have been numerous investigations into the underlying principle for CME differentiation and its sensitivity and specificity. ${ }^{12,15,37,38}$ Currently, TCD devices are rapidly increasing their diagnostic accuracy in differentiating between gaseous and solid CME and rejecting artifacts. Although the utilization of 2 different ultrasound frequencies allows TCD studies to be conducted with unprecedented accuracy. ${ }^{14}$ Together with the increasing number of reported neuroimaging and clinical studies related to CME evaluation, evidence is accruing that CME effectively represent Doppler-detectable correlates of microemboli. ${ }^{20,38-41}$ Thus, the technique used in the present study for CME detection and differentiation seems reliable. ${ }^{42}$

\section{Study Limitations}

One major limitation of this observational pilot study is the evaluation of a small patient cohort. Patients in the ICU typically have various surgical circumstances and medical diseases that might have potentially biased our results. Additional study confounders include anticoagulation, fluid therapy, hemodynamic state, and mechanical ventilation. We did not change patient positioning or treatment during TCD examinations in an attempt to best maintain steady state conditions. Ideally, however, it would have been beneficial to measure CME in a randomized fashion and also in the CVVHD circuit outlet to determine and correlate the number of microemboli that entered the venous circulation. Although differentiation between gaseous and solid CME can be performed accurately, technological algorithms can be false positive or negative in a few cases. ${ }^{12}$ Future CME studies should also consider ruling out the presence of an open foramen ovale. Although it was not incorporated into the present observational pilot trial, it would be advantageous to link patient CME burden to the modern neuroimaging evidence of silent brain infarction. Such studies would allow for the direct correlation of CME with morphologic signs of brain injury, thereby providing important information in support of the clinical significance of the present study's findings. Overall, the current findings should be regarded as representing a scientific perspective that needs further investigation and confirmation, and caution should be 
taken in extrapolating this evidence into clinical practice. We further would like to point out that it remains unknown and highly debated if gaseous or solid CME cause any brain injury at all.

\section{CONCLUSIONS}

This observational pilot study in critically ill patients demonstrated that activated CVVHD is associated with a higher gaseous CME burden. There were no differences in gaseous or solid CME counts between critically ill patients during deactivated CVVHD and a non-ICU control group of hospitalized patients before elective orthopedic surgery. These findings suggest that ICU therapy alone might not necessarily promote a higher CME count, but activated CVVHD might significantly increase CME burden in critically ill patients. The technology of automatic differentiation between gaseous and solid CME is challenging and the impact of the nature of $\mathrm{CME}$ on cerebral integrity remains unknown. However, this emerging field of silent brain infarction attributed to CME should be further investigated due to potentially high clinical relevance.

\section{ACKNOWLEDGEMENTS}

The authors greatly acknowledge the statistical support of Niels Hagenbuch, MD, MSc.

\section{REFERENCES}

1. Bischel MD, Scoles BG, Mohler JG. Evidence for pulmonary microembolization during hemodialysis. Chest. 1975;67:335-337.

2. Droste DW, Kuehne K, Schaefer RM, et al. Detection of microemboli in the subclavian vein of patients undergoing haemodialysis and haemodiafiltration using pulsed Doppler ultrasound. Nephrol Dial Transplant. 2002;17:462-466.

3. Rollé F, Pengloan J, Abazza M, et al. Identification of microemboli during haemodialysis using Doppler ultrasound. Nephrol Dial Transolant. 2000;15:1420-1424.

4. Woltmann D, Fatica RA, Rubin JM, et al. Ultrasound detection of microembolic signals in hemodialysis accesses. Am J Kindey Dis. 2000;35:526-528.

5. Droste DW, Beyna T, Trye B, et al. Reduction of circulating microemboli in the subclavian vein of patients undergoing haemodialysis using pre-filled instead of dry dialysers. Nephrol Dial Transplant. 2003;18:2377-2381.

6. Forsberg U, Jonsson P, Stegmayr C, et al. A high blood level in the air trap reduces microemboli during hemodialysis. Artif Organs. 2012;36:525-529.

7. Forsberg U, Jonsson P, Stegmayr C, et al. A high blood level in the venous chamber and a wet-stored dialyzer help to reduce exposure for microemboli during hemodialysis. Artif Organs. 2013;17: 612-617.

8. Barak M, Katz Y. Microbubbles: pathophysiology and clinical implications. Chest. 2005;128:2918-2932.

9. Forsberg U, Jonsson P, Stegmayr C, et al. Microemboli, developed during haemodialysis, pass the lung barrier and may cause ischaemic lesions in organs such as the brain. Nephrol Dial Transplant. 2010;25:2691-2695.

10. Madan P, Kalra OP, Agarwal S, et al. Cognitive impairment in chronic kidney disease. Nephrol Dial Transplant. 2007;22:440-444.

11. Darbellay GA, Duff R, Vesin J-M, et al. Solid or gaseous circulating brain emboli: are they separable by transcranial ultrasound? J Cereb Blood Flow Metab. 2004;24:860-868.

12. Devuyst G, Darbellay GA, Vesin JM, et al. Automatic classification of HITS into artifacts or solid or gaseous emboli by a wavelet representation combined with dual-gate TCD. Stroke. 2001;32: 2803-2809.

13. Abu-Omar Y, Balacumaraswami L, Pigott DW, et al. Solid and gaseous cerebral microembolization during off-pump, on-pump, and open cardiac surgery procedures. $J$ Thorac Cardiovasc Surg. 2004:127:1759-1765.

14. Brucher R, Russel D. Automatic online embolus detection and artifact rejection with the first multifrequency transcranial Doppler. Stroke. 2002;33:1969-1974.

15. Russel D, Brucher R. Online automatic discrimination between solid and gaseous cerebral microemboli with the first multifrequency transcranial Doppler. Stroke. 2002;33:1975-1980.

16. Guerrieri Wolf L, Abu-Omar Y, Choudhary BP, et al. Gaseous and solid cerebral microembolization during proximal aortic anastomoses in off-pump coronary surgery: the effect of an aortic sidebiting clamp and two clampless devices. $J$ Thorac Cardiovasc Surg. 2007; 133:485-493.

17. Guerrieri Wolf L, Choudhary BP, Abu-Omar Y, et al. Solid and gaseous cerebral microembolization after biologic and mechanical aortic valve replacement: investigation with multirange and multifrequency transcranial Doppler ultrasound. J Thorac Cardiovasc Surg. 2008;135:512-520.

18. Maselli D, Pizio R, Musumeci F. Multifrequency transcranial Doppler for intraoperative automatic detection and characterisation of cerebral microemboli during port-access mitral valve surgery. Interact Cardiovasc Thorac Surg. 2006;5:32-35.

19. Rodriguez RA, Nathan HJ, Ruel M, et al. A method to distinguish between gaseous and solid cerebral emboli in patients with prosthetic heart valves. Eur J Cardiothorac Surg. 2009;35:89-95.

20. Skjelland M, Krohg-Sørensen K, Tennøe B, et al. Cerebral microemboli and brain injury during carotid artery endarterectomy and stenting. Stroke. 2009;40:230-234.

21. Ringelstein EB, Droste DW, Babikian VL, et al. Consensus on microembolus detection by TCD. International Consensus Group on Microembolus Detection. Stroke. 1998;29:725-729.

22. Jonsson P, Karlsson L, Forsberg U, et al. Air bubbles pass the security system of the dialysis device without alarming. Artif Organs. 2007;31:132-139.

23. Bedell E, Berge KH, Losasso TJ, et al. Paradoxic air embolism during venous air embolism: transesophageal echocardiographic evidence of transpulmonary air passage. Anesthesiology. 1994;80: $947-950$

24. George S, Holt S, Hildrick-Smith D. Patent foramen ovale, dialysis and microembolization. Nephrology. 2012;17:569-574.

25. Orlandi G, Parenti G, Bertolucci A, et al. Silent cerebral microembolism in asymptomatic and symptomatic carotid artery stenoses of low and high degree. Eur Neurol. 1997;38:39-43.

26. Goertler M, Baeumer M, Kross R, et al. Rapid decline of cerebral microemboli of arterial origin after intravenous acetylsalicylic acid. Stroke. 1999;30:66-69.

27. Rundek T, Di Tullio MR, Sciacca RR, et al. Association between large aortic arch atheromas and high-intensity transient signals in elderly stroke patients. Stroke. 1999;30:2683-2686.

28. Nadareishvili ZG, Choudary Z, Joyner C, et al. Cerebral microembolism in acute myocardial infarction. Stroke. 1999;30: 2679-2682.

29. Goldberg I, Auriel E, Russell D, et al. Microembolism, silent brain infarcts and dementia. J Neurol Sci. 2012;322:250-253.

30. Stroobant N, Van Nooten G, De Bacquer D, et al. Neuropsychological functioning 3-5 years after coronary artery bypass grafting: does the pump make a difference? Eur J Cardiothorac Surg. 2008;34:396-401.

31. Groom RC, Quinn RD, Lennon P, et al. Detection and elimination of microemboli related to cardiopulmonary bypass. Circ Cardiovasc Qual Outcomes. 2009;2:191-198.

32. Caplan LR, Hennerici M. Impaired clearance of emboli (washout) is an important link between hypoperfusion, embolism, and ischemic stroke. Arch Neurol. 1998;55:1475-1482.

33. Fazekas G, Fazekas F, Schmidt R, et al. Brain MRI findings and cognitive impairment in patients undergoing chronic hemodialysis treatment. J Neurol Sci. 1995;134:83-88. 
34. Savazzi GM, Cusmano F, Musini S. Cerebral imaging changes in patients with chronic renal failure treated conservatively or in hemodialysis. Nephron. 2001;89:31-36.

35. Kamata T, Hishida A, Takita T, et al. Morphologic abnormalities in the brain of chronically hemodialyzed patients without cerebrovascular disease. Am J Nephrol. 2000;20:27-31.

36. Murray AM, Tupper DE, Knopman DS, et al. Cognitive impairment in hemodialysis patients is common. Neurology. 2006;67: 216-223.

37. Markus HS, Brown MM. Differentiation between different pathological cerebral embolic materials using transcranial Doppler in an in vitro model. Stroke. 1993;24:1-5.

38. Ackerstaff RG, Moons KG, van de Vlasakker CJ, et al. Association of intraoperative transcranial Doppler monitoring variables with stroke from carotid endarterectomy. Stroke. 2000;31:1817-1823.
39. Abu-Omar Y, Cader S, Guerrieri Wolf L, et al. Short-term changes in cerebral activity in on-pump and off-pump cardiac surgery defined by functional magnetic resonance imaging and their relationship to microembolization. J Thorac Cardiovasc Surg. 2006; 132:1119-1125.

40. Liu YH, Wang DX, Li LH, et al. The effects of cardiopulmonary bypass on the number of cerebral microemboli and the incidence of cognitive dysfunction after coronary artery bypass graft surgery. Anesth Analg. 2009;109:1013-1022.

41. Gerriets T, Schwarz N, Sammer G, et al. Protecting the brain from gaseous and solid micro-emboli during coronary artery bypass grafting: a randomized controlled trial. Eur Heart J. 2010;31: 360-368.

42. Ainslie PN, Hoiland RL. Transcranial Doppler ultrasound: valid, invalid, or both? J Appl Physiol. 2014;117:1081-1083. 